\title{
ON ROBUST STABILITY OF UNCERTAIN NEUTRAL SYSTEMS WITH TIME-VARYING DISCRETE DISTRIBUTED DELAYS
}

\author{
Q.-L. Han \\ Faculty of Informatics and Communication \\ Central Queensland University \\ Rockhampton, Qld 4702, AUSTRALIA \\ Tel: + 61749309270 \\ Fax: + 61749309729 \\ E-mail: q.hanecqu.edu.au
}

Keywords: Stability, discrete delay, distributed delay, neutral system, linear matrix inequality (LMI).

\begin{abstract}
The robust stability of uncertain linear neutral systems with time-varying discrete and distributed delays is investigated by employing descriptor model transformation and the decomposition technique of discrete-delay term matrix. The proposed delay-dependent stability criteria are formulated in the form of a linear matrix inequality. Numerical examples are given to indicate significant improvements over some existing results.
\end{abstract}

\section{Introduction}

The problem of stability of time-delay systems of neutral type has received considerable attention in the last two decades; see for example [7]. Current efforts on this topic can be divided into two categories, namely delayindependent stability criteria and delay-dependent stability criteria.

For linear neutral systems with constant delays, some delay-independent stability conditions were obtained. They were formulated in terms of a matrix measure and a matrix norm [11] or the existence of a positive definite solution to an auxiliary algebraic Riccati matrix equation $[16,17]$. Although it is easy to check these conditions, the conditions required the matrix measure to be negative or the parameters to be tuned.

A model transformation technique is often used to transform the neutral system with constant discrete delay to a neutral system with a constant distributed delay, and delay-dependent stability criteria are obtained by employing Lyapunov-Krasovskii functionals [8, 9, 10, 13, 14]. These results are usually less conservative than the delay-independent stability ones. Some of these results are still conservative since some model transformations will introduce additional dynamics discussed in $[5,6]$.

Recently, a new descriptor model transformation and a corresponding Lyapunov-Krasovskii functional have been introduced for stability analysis of systems with constant delays in [1]. The advantage of this transformation is to transform the original system to an equivalent descriptor form representation and will not introduce additional dynamics in the sense defined in [5, 6]. In [2], some results in [1] are extended to neutral systems with timevarying discrete delays. Although the result in [2] is less conservative than some existing ones, it can be improved by employing the decomposition technique to get a larger bound for time-varying discrete delays. Furthermore, timevarying distributed delays and uncertainties are not considered in [2].

In this paper, based on the descriptor model transformation and the decomposition technique of discrete-delay term matrix, we will investigate the robust stability of uncertain neutral systems with time-varying discrete and distributed delays by applying an integral inequality that is introduced in this paper instead of applying bounding of the cross terms introduced in [15]. The robust stability problem of considered system will be transformed into the existence of some symmetric positive-definite matrices. The stability criteria will be formulated in the form of linear matrix inequalities (LMIs). Numerical examples will show that the results obtained in this paper are less conservative than some existing ones in the literature. 


\section{Problem statement}

Consider the following linear neutral system with timevarying discrete and distributed delays

$$
\begin{aligned}
\dot{x}(t)-C \dot{x}(t-\tau)= & A(t) x(t)+B(t) x(t-h(t)) \\
& +D(t) \int_{t-r(t)}^{t} x(\xi) d \xi
\end{aligned}
$$

where $x(t) \in \mathbb{R}^{n}$ is the state, $C \in \mathbb{R}^{n \times n}$ is a known constant matrix, and $A(t) \in \mathbb{R}^{n \times n}, \quad B(t) \in \mathbb{R}^{n \times n}$, and $D(t) \in \mathbb{R}^{n \times n} \quad$ are uncertain matrices, not known completely, except that they are within a compact set $\Omega$ which we will refer to as the uncertainty set

$$
(A(t), B(t), D(t)) \in \Omega \subset \mathbb{R}^{n \times 3 n} \text { for all } t \in[0, \infty)
$$

and $\tau \geq 0$ is a constant neutral delay, $h(t)$ is a timevarying discrete delay and $r(t)$ is a time-varying distributed delay, which satisfy

$$
0 \leq h(t) \leq h_{M}, \dot{h}(t) \leq h_{d} ; 0 \leq r(t) \leq r_{M}, \dot{r}(t) \leq r_{d}
$$

where $h_{M}, h_{d}, r_{M}$ and $r_{d}$ are constants, and $0 \leq h_{d}<1$ and $0 \leq r_{d}<1$.

The initial condition of system (1) is given by

$$
x\left(t_{0}+\theta\right)=\varphi(\theta), \forall \theta \in\left[-\max \left\{\tau, h_{M}, r_{M}\right\}, 0\right]
$$

where $\varphi(\cdot)$ is a continuous vector valued initial function.

The purpose of this paper is to formulate some practically computable criteria to check the robust stability of system described by (1) (4).

\section{Main results}

Rewrite system (1) in the following equivalent descriptor system

$$
\begin{aligned}
\dot{x}(t)= & y(t) \\
y(t)= & C y(t-\tau)+A(t) x(t)+B(t) x(t-h) \\
& +D(t) \int_{t-r}^{t} x(\xi) d \xi
\end{aligned}
$$

To derive discrete-delay-dependent stability conditions, which include the information of the time-delay $h(t)$, one usually uses the fact

$$
x(t-h(t))=x(t)-\int_{t-h(t)}^{t} \dot{x}(\xi) d \xi=x(t)-\int_{t-h(t)}^{t} y(\xi) d \xi
$$

to transform the original system (1) to a system with a distributed delay. This transformation does not provide any additional dynamics because we do not expand $\dot{x}(t)$ in terms of right hand representation of (1).

In order to improve the bound of discrete-delay $h(t)$, let's decompose the matrix $B(t)$ as

$$
B(t)=B_{1}+B_{2}(t)
$$

where $B_{1}$ is a constant matrix. Then system (5) can be represented in the form of descriptor system with discrete and distributed delays in the "fast variable" $y[1]$

$$
\begin{aligned}
\dot{x}(t) & =y(t) \\
0= & -y(t)+C y(t-\tau)+\left(A(t)+B_{1}\right) x(t)+B_{2}(t) x(t-h(t)) \\
& -B_{1} \int_{t-h(t)}^{t} y(\xi) d \xi+D(t) \int_{t-r(t)}^{t} x(\xi) d \xi
\end{aligned}
$$

Noting that system (6) is equivalent to system (1), in the following we will employ system (6) to study the stability of system (1).

Throughout this paper, we assume that

A1. All the eigenvalues of matrix $C$ are inside the unit circle.

For robust stability of system (1) (4), we have the following result.

Proposition 1. Under A1, the system described by (1) to (4) is asymptotically stable if there exist real matrices $P_{2}, P_{3}$ and symmetric positive definite matrices $P_{1}, Q$, $R, S$, and $W$ such that the LMI (7), as shown at the bottom of the last page of the paper, holds, where

$$
\begin{aligned}
& \Xi_{11}(t)=P_{2}^{T}\left(A(t)+B_{1}\right)+\left(A(t)+B_{1}\right)^{T} P_{2}+Q+r_{M} W \\
& \Xi_{12}(t)=P_{1}-P_{2}^{T}+\left(A(t)+B_{1}\right)^{T} P_{3} \\
& \Xi_{22}(t)=-P_{3}-P_{3}^{T}+R+h_{M} S .
\end{aligned}
$$

Choose the Lyapunov-Krasovskii functional candidate for system (6) as

$$
V(t)=V_{1}(t)+V_{2}(t)+V_{3}(t)+V_{4}(t)+V_{5}(t)
$$

where

$$
\begin{aligned}
& V_{1}(t)=\left(x^{T}(t) y^{T}(t)\right)\left[\begin{array}{ll}
I & 0 \\
0 & 0
\end{array}\right]\left[\begin{array}{ll}
P_{1} & 0 \\
P_{2} & P_{3}
\end{array}\right]\left(\begin{array}{l}
x(t) \\
y(t)
\end{array}\right) \\
& V_{2}(t)=\int_{t-h(t)}^{t} x^{T}(\xi) Q x(\xi) d \xi
\end{aligned}
$$




$$
\begin{aligned}
& V_{3}(t)=\int_{t-\tau}^{t} y^{T}(\xi) R y(\xi) d \xi \\
& V_{4}(t)=\int_{t-h(t)}^{t}(h(t)-t+\xi) y^{T}(\xi) S y(\xi) d \xi \\
& V_{5}(t)=\int_{t-r(t)}^{t}(r(t)-t+\xi) x^{T}(\xi) W x(\xi) d \xi
\end{aligned}
$$

where $P_{2}, P_{3}$ and symmetric positive definite matrices $P_{1}$, $Q, R, S$ and $W$ are the solutions of (7). One can prove Proposition 1. For the detail, see the full version of the paper [10].

To compare with some existing results, now we consider the nominal system of system (1) with constant discrete and distributed delays, that is

$$
\dot{x}(t)-C \dot{x}(t-\tau)=A x(t)+B x(t-h)+D \int_{t-r}^{t} x(\xi) d \xi
$$

with the initial condition

$$
x\left(t_{0}+\theta\right)=\varphi(\theta), \forall \theta \in[-\max \{\tau, h, r\}, 0]
$$

By Proposition 1, we have the following corollary

Corollary 1. Under A1, the system described by (8) and (9) is asymptotically stable if there exist real matrices $P_{2}, P_{3}$ and symmetric positive definite matrices $P_{1}, Q, R, S$, and $W$ such that the following LMI holds

$\left[\begin{array}{cccccc}\Xi_{11} & \Xi_{12} & P_{2}^{T} B_{2} & P_{2}^{T} C & -h P_{2}^{T} B_{1} & r P_{2}^{T} D \\ \Xi_{12}^{T} & \Xi_{22} & P_{3}^{T} B_{2} & P_{3}^{T} C & -h P_{3}^{T} B_{1} & r P_{3}^{T} D \\ B_{2}^{T} P_{2} & B_{2}^{T} P_{3} & -Q & 0 & 0 & 0 \\ C^{T} P_{2} & C^{T} P_{3} & 0 & -R & 0 & 0 \\ -h B_{1}^{T} P_{2} & -h B_{1}^{T} P_{3} & 0 & 0 & -h S & 0 \\ r D^{T} P_{2} & r D^{T} P_{3} & 0 & 0 & 0 & -r W\end{array}\right]<0 \quad(10)$

where

$$
\begin{aligned}
& \Xi_{11}=P_{2}^{T}\left(A+B_{1}\right)+\left(A+B_{1}\right)^{T} P_{2}+Q+r W \\
& \Xi_{12}=P_{1}-P_{2}^{T}+\left(A+B_{1}\right)^{T} P_{3} \\
& \Xi_{22}=-P_{3}-P_{3}^{T}+R+h S
\end{aligned}
$$

Remark 1. It is clear that condition (10) guarantees that system (8) with (9) is of delay-independent stability with respect to the neutral delay $\tau \geq 0$.
Remark 2. If $B_{2}=0$, then $B_{1}=B$. In this case, (10) implies

$$
\left[\begin{array}{ccccc}
(1,1) & (1,2) & P_{2}^{T} C & -h P_{2}^{T} B & r P_{2}^{T} D \\
(1,2)^{T} & (2,2) & P_{3}^{T} C & -h P_{3}^{T} B & r P_{3}^{T} D \\
C^{T} P_{2} & C^{T} P_{3} & -R & 0 & 0 \\
-h B^{T} P_{2} & -h B^{T} P_{3} & 0 & -h S & 0 \\
r D^{T} P_{2} & r D^{T} P_{3} & 0 & 0 & -r W
\end{array}\right]<0
$$

with

$$
\begin{aligned}
& (1,1) \triangleq(A+B)^{T} P_{2}+P_{2}^{T}(A+B)+r W \\
& (1,2) \triangleq P_{1}-P_{2}^{T}+(A+B)^{T} P_{3} \\
& (2,2) \triangleq-P_{3}-P_{3}^{T}+R+h S
\end{aligned}
$$

which is (26) in Theorem 2 in [1] for the case of $k=1$, $m=1$ and $F_{1}=0$.

Remark 3. If $B_{1}=0$, then $B_{2}=B$. The LMI (10) implies

$$
\left[\begin{array}{ccccc}
(1,1) & (1,2) & P_{2}^{T} C & P_{2}^{T} B & r P_{2}^{T} D \\
(1,2)^{T} & (2,2) & P_{3}^{T} C & P_{3}^{T} B & r P_{3}^{T} D \\
C^{T} P_{2} & C^{T} P_{3} & -R & 0 & 0 \\
B^{T} P_{2} & B^{T} P_{3} & 0 & -Q & 0 \\
r D^{T} P_{2} & r D^{T} P_{3} & 0 & 0 & -r W
\end{array}\right]<0
$$

with

$$
\begin{aligned}
& (1,1) \triangleq A^{T} P_{2}+P_{2}^{T} A+Q+r W \\
& (1,2) \triangleq P_{1}-P_{2}^{T}+A^{T} P_{3} \\
& (2,2) \triangleq-P_{3}-P_{3}^{T}+R
\end{aligned}
$$

which recovers (29) in Corollary 2 in [1] for the case of $k=1, m=1$ and $F_{1}=0$. The LMI above and A1 are neutral-delay-independent, discrete-delay-independent and distributed-delay-dependent conditions for system (8) with (9) to be asymptotically stable.

Remark 4. If $\tau=h, D=0$ and $B_{2}=0$, then (10) implies

$$
\left[\begin{array}{cccc}
(A+B)^{T} P_{2}+P_{2}^{T}(A+B) & P_{1}-P_{2}^{T}+(A+B)^{T} P_{3} & P_{2}^{T} C & -h P_{2}^{T} B \\
P_{1}^{T}-P_{2}+P_{3}^{T}(A+B) & -P_{3}-P_{3}^{T}+R+h S & P_{3}^{T} C & -h P_{3}^{T} B \\
C^{T} P_{2} & C^{T} P_{3} & -R & 0 \\
-h B^{T} P_{2} & -h B^{T} P_{3} & 0 & -h S
\end{array}\right]<0
$$

which is (8) in Theorem 1 in [1] for the case of $m=1$.

Remark 5. If $\tau=h, D=0$ and $B_{1}=0$, then (10) implies 


$$
\left[\begin{array}{cccc}
A^{T} P_{2}+P_{2}^{T} A+Q & P_{1}-P_{2}^{T}+A^{T} P_{3} & P_{2}^{T} C & P_{2}^{T} B \\
P_{1}^{T}-P_{2}+P_{3}^{T} A & -P_{3}-P_{3}^{T}+R & P_{3}^{T} C & P_{3}^{T} B \\
C^{T} P_{2} & C^{T} P_{3} & -R & 0 \\
B^{T} P_{2} & B^{T} P_{3} & 0 & -Q
\end{array}\right]<0
$$

which together with A1 is a sufficient condition for delayindependent stability for system (8) with (9) (without a distributed term).

Remark 6. From the discussions above, it is clear to see that Corollary 1 establishes a "bridge" between discretedelay-independent stability and discrete-delay-dependent stability for system (8) with (9).

Remark 7. The efficiency of Proposition 1 and Corollary 1 depends on the decomposition of matrix $B$. The matrix $B_{1}$ is chosen such that $A+B_{1}$ is "more stable" than matrix $A$. The decomposition idea was firstly introduced by Goubet-Batholomeus et al. (1997) [4] for the retarded case. Now we consider how to decompose the matrix $B$. For the sake of simplicity, we take Corollary 1 as an example and restrict $Q, S$, and to a special case of $Q=S=P_{1}$. From (10), one can see that $-P_{3}-P_{3}^{T}<0$. So the matrix $\left[\begin{array}{cc}P_{1} & 0 \\ P_{2} & P_{3}\end{array}\right]$ is nonsingular. Define $\left[\begin{array}{ll}P_{1} & 0 \\ P_{2} & P_{3}\end{array}\right]^{-1}=\left[\begin{array}{cc}X_{1} & 0 \\ X_{2} & X_{3}\end{array}\right]$ and $K=\operatorname{diag}\{X, I, I, I, I\}$.

Then we multiply (10) by $K^{T}$ and $K$, on the left and right, respectively. Using the Shur's complement to the quadratic term in $K$, and introducing new variables $Y=R^{-1}, Z=W^{-1}$ and $L=B_{1} X_{1}$ to yield the following LMI

$$
\left(\begin{array}{ll}
\Gamma_{11} & \Gamma_{12} \\
\Gamma_{12}^{T} & \Gamma_{22}
\end{array}\right)<0
$$

where

$$
\begin{aligned}
\Gamma_{11} & =\left(\begin{array}{ccccc}
X_{2}+X_{2}^{T} & -X_{2}^{T}+X_{1} A^{T}+L^{T} \\
-X_{2}+A X_{1}+L & -X_{3}-X_{3}^{T}
\end{array}\right) \\
\Gamma_{12} & =\left(\begin{array}{cccccccc}
0 & 0 & 0 & 0 & X_{1} & h X_{2}^{T} & r X_{1} & X_{2}^{T} \\
B X_{1} & C Y & -h L & r D Z & 0 & h X_{3}^{T} & 0 & X_{3}^{T}
\end{array}\right) \\
\Gamma_{22} & =\operatorname{diag}\left\{-X_{1},-Y,-h X_{1},-r Z,-X_{1},-h X_{1},-r Z,-Y\right\}
\end{aligned}
$$

Then we can conclude that under A1, the system described by (8) and (9) is asymptotically stable if there exist real matrices $X_{2}, X_{3}, L$, and symmetric positive definite matrices $X_{1}, Y$ and $Z$ such that the LMI (11) holds.
Furthermore, the matrix $B_{1}$ is given by $B_{1}=L X_{1}^{-1}$. For the general case, the idea is the same. It is omitted.

For a polytopic uncertainty, it is clearly sufficient that (7) only needs to be satisfied at all the vertices. Now we consider the norm bounded uncertainty described by

$A(t)=A+\Delta A(t), B(t)=B+\Delta B(t), D(t)=D+\Delta D(t)(12)$

where

$$
[\Delta A(t) \Delta B(t) \Delta D(t)]=L F(t)\left[\begin{array}{lll}
E_{a} & E_{b} & E_{d}
\end{array}\right]
$$

where $F(t) \in \mathbb{R}^{p \times q}$ is an unknown real and possibly timevarying matrix with Lebesgue measurable elements satisfying

$$
\sigma_{\max }(F(t)) \leq 1
$$

and $L, E_{a}, E_{b}$, and $E_{d}$ are known real constant matrices which characterize how the uncertainty enters the nominal matrices $A, B$, and $D$.

Let $B=B_{1}+B_{2}$, then $B_{2}(t)=B_{2}+\Delta B(t)$. Now we state the following result.

Proposition 2. Under A1, the system described by (1), (3) and (4), with uncertainty described by (12) to (14) is asymptotically stable if there exist real matrices $\tilde{P}_{2}, \tilde{P}_{3}$ and symmetric positive definite matrices $\tilde{P}_{1}, \tilde{Q}, \tilde{R}, \tilde{S}$, and $\tilde{W}$, such that the LMI (15), as shown at the bottom of the last page of the paper, holds, where

$$
\begin{aligned}
& \tilde{\Xi}_{11}^{0}=\tilde{P}_{2}^{T}\left(A+B_{1}\right)+\left(A+B_{1}\right)^{T} \tilde{P}_{2}+\tilde{Q}+r_{M} \tilde{W} \\
& \tilde{\Xi}_{12}^{0}=\tilde{P}_{1}-\tilde{P}_{2}^{T}+\left(A+B_{1}\right)^{T} \tilde{P}_{3} \\
& \tilde{\Xi}_{22}^{0}=-\tilde{P}_{3}-\tilde{P}_{3}^{T}+\tilde{R}+h_{M} \tilde{S}
\end{aligned}
$$

\section{Examples}

In order to use Proposition 2 to test the stability of the system described by (1), (3) and (4), with uncertainty described by (12) to (14), a MATLAB m-function is written which automatically generates LMI (15), and then solves this LMI using LMI Solver FEASP in MATLAB LMI toolbox [3]. The inputs to the function are system matrices and time-delays. The function returns whether the LMI is feasible. If feasible, it also gives matrices real matrices $\tilde{P}_{2}, \tilde{P}_{3}$ and symmetric positive definite matrices $\tilde{P}_{1}, \tilde{Q}, \tilde{R}, \tilde{S}$ and $\tilde{W}$ as the outputs. The following examples are generated using this MATLAB m-function. 
Example 1. Consider the following uncertain neutral system with time-varying discrete delay

$$
\begin{aligned}
\dot{x}(t)= & \left(\begin{array}{cc}
-2+\delta_{1} \cos (t) & 0 \\
0 & -1+\delta_{2} \sin (t)
\end{array}\right) x(t) \\
& +\left(\begin{array}{cc}
-1+\gamma_{1} \cos (t) & 0 \\
-1 & -1+\gamma_{2} \sin (t)
\end{array}\right) x(t-h(t))
\end{aligned}
$$

where $0 \leq|c|<1$ and $\delta_{1}, \delta_{2}, \gamma_{1}$ and $\gamma_{2}$ are unknown parameters satisfying

$$
\left|\delta_{1}\right| \leq 1.6,\left|\delta_{2}\right| \leq 0.05,\left|\gamma_{1}\right| \leq 0.1,\left|\gamma_{2}\right| \leq 0.3
$$

For $h_{d}=0$, the maximum value of $h_{M}$ for stability of system (16) was reported as $h_{M}=0.2412$ and $h_{M}=1.0$ in [12] and [2], respectively. Now we use the criterion in this paper to study the same case. Let's decompose the matrix $B$ as $B=B_{1}+B_{2}$, where

$$
B_{1}=\left[\begin{array}{cc}
-0.47 & 0 \\
-0.01 & -0.58
\end{array}\right], B_{2}=\left[\begin{array}{cc}
-0.53 & 0 \\
-0.99 & -0.42
\end{array}\right]
$$

the maximum value of $h_{M}$ for the system to be asymptotically stable is $h_{M}=1.1285$. This example shows that the stability criterion in this paper gives a much less conservative result than these in $[12,2]$.

Example 2. Consider the following uncertain neutral delay-differential system

$$
\begin{aligned}
\dot{x}(t)-C \dot{x}(t-\tau)= & (A+\Delta A(t)) x(t) \\
& +(B+\Delta B(t)) x(t-h(t))
\end{aligned}
$$

where

$$
A=\left(\begin{array}{cc}
-0.9 & 0.2 \\
0.1 & -0.9
\end{array}\right), B=\left(\begin{array}{cc}
-1.1 & -0.2 \\
-0.1 & -1.1
\end{array}\right), C=\left(\begin{array}{cc}
-0.2 & 0 \\
0.2 & -0.1
\end{array}\right)
$$

and $\Delta A(t)$ and $\Delta B(t)$ are unknown matrices satisfying $\|\Delta A(t)\| \leq \alpha$ and $\|\Delta B(t)\| \leq \alpha, \quad \alpha \geq 0, \forall t$. The above system is of the form of (12) to (14) with $L=\alpha I$ and $E_{a}=E_{b}=I$.

Decompose matrix $B$ as $B=B_{1}+B_{2}$, where

$$
B_{1}=\left[\begin{array}{ll}
-0.34 & -0.06 \\
-0.02 & -0.35
\end{array}\right], B_{2}=\left[\begin{array}{ll}
-0.76 & -0.14 \\
-0.08 & -0.75
\end{array}\right]
$$

For the nominal system, i.e. $\Delta A(t) \equiv 0$ and $\Delta B(t) \equiv 0$, with $h(t) \equiv h=$ const (i.e. $h_{d} \equiv 0$ ) and $\tau=h$, system (23) reduces to the system discussed in [13]. Using the criterion in this paper, the maximum value of $h_{M}$ for the nominal system to be asymptotically stable is $h_{M}=1.5758$. By the criteria in $[13,14,1]$ the nominal system is asymptotically stable for any $h$ satisfying $h \leq 0.3, h \leq 0.71$, and $h \leq 0.74$, respectively. This example shows that the stability criterion in this paper gives a much less conservative result than these in $[13,14,1]$.

For $\alpha=0.2$, the following table gives different $h_{M}$ for different $h_{d}$. It is clear that as $h_{d}$ increases, the corresponding $h_{M}$ decreases.

\begin{tabular}{|c|c|c|c|}
\hline$h_{d}$ & 0.00 & 0.05 & 0.10 \\
\hline$h_{M}$ & 1.1586 & 1.0610 & 0.9557 \\
\hline$h_{d}$ & 0.15 & 0.20 & 0.25 \\
\hline$h_{M}$ & 0.8406 & 0.7126 & 0.5658 \\
\hline
\end{tabular}

For $h_{d}=0.1$, the effect of the uncertainty bound $\alpha$ on the maximum time-delay for stability $h_{M}$ is also studied. The following table illustrates the numerical results for different $\alpha$. We can see that as $\alpha \rightarrow 0$, the stability limit for delay approaches the uncertainty-free case. As $\alpha$ increases, $h_{M}$ decreases.

\begin{tabular}{|c|c|c|c|c|}
\hline$h_{d}$ & 0.00 & 0.05 & 0.10 & 0.15 \\
\hline$h_{M}$ & 1.3797 & 1.2792 & 1.1750 & 1.0615 \\
\hline$h_{d}$ & 0.20 & 0.25 & 0.30 & 0.35 \\
\hline$h_{M}$ & 0.9557 & 0.8404 & 0.7213 & 0.5980 \\
\hline
\end{tabular}

\section{Conclusion}

A delay-dependent stability criterion for neutral systems with time-varying discrete delay has been obtained. The criterion has been expressed in the form of a linear matrix inequality (LMI). Numerical examples have shown that the results derived by criterion in this paper are much less conservative than some existing ones in the literature.

\section{References}

[1] E. Fridman. "New Lyapunov-Krasovskii functionals for stability of linear retarded and neutral type systems," Systems \& Control Letters, 43, 309 319, (2001). 
[2] E. Fridman, U. Shaked. "Stability and $H_{\infty}$ control of systems with time-varying delays," In preprint Proc. of $15^{\text {th }}$ IFAC World Congress (CD), Barcelona, Spain (2002).

[3] P. Gahinet, A. Nemirovski, A. J. Laub, M. Chilali. LMI Control Toolbox: For use with MATLAB, Natick, MA: Math Works (1995).

[4] A. Goubet-Batholomeus, M. Dambrine, J. P. Richard. "Stability of perturbed systems with time-varying delays," Syst. Contr. Lett., 31, pp. 155-163 (1997).

[5] K. Gu, S.-I. Niculescu. "Additional dynamics in transformed time-delay systems," IEEE Trans. Automat. Contr., 45, 572-575 (2000).

[6] K. Gu, S.-I. Niculescu. "Further remarks on additional dynamics in various model transformations of linear delay systems," IEEE Trans. Automat. Contr., 46, 497-500 (2001).

[7] J. K. Hale, S. M. Verduyn Lunel. Introduction to Functional Differential Equation, New York, SpringerVerlag (1993).

[8] Q.-L. Han. “On delay-dependent stability for neutral delay-differential systems," Int. J. Appl. Math. and Comp. Sci., 11, 965-976 (2001).

[9] Q.-L. Han. "Robust stability of uncertain delay-differential systems of neutral type," Automatica, 38, 719-723 (2002).

[10] Q.-L. Han. "On robust stability of uncertain neutral systems with time-varying discrete and distributed delays,"
Internal Report, Central Queesland University (2002).

[11] G. Di Hu, G. Da Hu. "Some simple stability criteria of neutral delay-differential systems," Appl. Math. Comput. 80, 257-271 (1996).

[12] J.-H. Kim. "Delay and its time-derivative dependent robust stability of time-delayed linear systems with uncertainty," IEEE Transactions on Automatic Control, 46, 789-792 (2001).

[13] C.-H. Lien, K.-W. Yu, J.-G. Hsieh. "Stability conditions for a class of neutral systems with multiple time delays," Journal of Mathematical Analysis and Applications, 245, 20-27 (2000).

[14] S.-I. Niculescu. "Further remarks on delay-dependent stability of linear neutral systems," In Proc. of MTNS 2000, Perpigan, France (2002).

[15] P. Park. "A delay-dependent stability criterion for systems with uncertain time-invariant delays," IEEE Trans. Automat. Contr, 44, 876-877 (1999).

[16] M. Slemrod, E. F. Infante. "Asymptotic stability criteria for linear systems of differential equations of neutral type and their discrete analogues," J. Math. Anal. Appl., 38, 399-415 (1972).

[17] E.-I. Verriest, S.-I. Niculescu. "Delay-independent stability of linear neutral systems: A Riccati equation approach," in Stability and Control of Time-delay Systems (L. Dugard and E. I. Verriest, Eds.) LNCIS, Vol. 228, Springer-Verlag, London, pp. 92-100 (1997).

$$
\Xi(t)=\left[\begin{array}{cccccc}
\Xi_{11}(t) & \Xi_{12}(t) & P_{2}^{T} B_{2}(t) & P_{2}^{T} C & -h_{M} P_{2}^{T} B_{1} & r_{M} P_{2}^{T} D(t) \\
\Xi_{12}^{T}(t) & \Xi_{22}(t) & P_{3}^{T} B_{2}(t) & P_{3}^{T} C & -h_{M} P_{3}^{T} B_{1} & r_{M} P_{3}^{T} D(t) \\
B_{2}^{T}(t) P_{2} & B_{2}^{T}(t) P_{3} & -\left(1-h_{d}\right) Q & 0 & 0 & 0 \\
C^{T} P_{2} & C^{T} P_{3} & 0 & -R & 0 & 0 \\
-h_{M} B_{1}^{T} P_{2} & -h_{M} B_{1}^{T} P_{3} & 0 & 0 & -\left(1-h_{d}\right) h_{M} S & 0 \\
r_{M} D^{T}(t) P_{2} & r_{M} D^{T}(t) P_{3} & 0 & 0 & 0 & -\left(1-r_{d}\right) r_{M} W
\end{array}\right]<0
$$

$\left[\begin{array}{cccccccc}\tilde{\Xi}_{11}^{0} & \tilde{\Xi}_{12}^{0} & \tilde{P}_{2}^{T} B_{2} & \tilde{P}_{2}^{T} C & -h_{M} \tilde{P}_{2}^{T} B_{1} & r_{M} \tilde{P}_{2}^{T} D & \tilde{P}_{2}^{T} L & E_{a}^{T} \\ \tilde{\Xi}_{12}^{0 T} & \tilde{\Xi}_{22}^{0} & \tilde{P}_{3}^{T} B_{2} & \tilde{P}_{3}^{T} C & -h_{M} \tilde{P}_{3}^{T} B_{1} & r_{M} \tilde{P}_{3}^{T} D & \tilde{P}_{3}^{T} L & 0 \\ B_{2}^{T} \tilde{P}_{2} & B_{2}^{T} \tilde{P}_{3} & -\left(1-h_{d}\right) \tilde{Q} & 0 & 0 & 0 & 0 & E_{b}^{T} \\ C^{T} \tilde{P}_{2} & C^{T} P_{3} & 0 & -\tilde{R} & 0 & 0 & 0 & 0 \\ -h_{M} B_{1}^{T} \tilde{P}_{2} & -h_{M} B_{1}^{T} \tilde{P}_{3} & 0 & 0 & -\left(1-h_{d}\right) h_{M} \tilde{S} & 0 & 0 & 0 \\ r_{M} D^{T} \tilde{P}_{2} & r_{M} D^{T} \tilde{P}_{3} & 0 & 0 & 0 & -\left(1-r_{d}\right) r_{M} \tilde{W} & 0 & r_{M} E_{d}^{T} \\ L^{T} \tilde{P}_{2} & L^{T} \tilde{P}_{3} & 0 & 0 & 0 & 0 & -I & 0 \\ E_{a} & 0 & E_{b} & 0 & 0 & 0 & 0 & -I\end{array}\right]<0$

